\title{
LETTER FROM THE EDITOR September 2021
}

In the editorial section of this issue, we have two columns to share. The September Editorial Board Thoughts essay is by Paul Swanson, "Building a Culture of Resilience in Libraries," reflecting on the lessons of COVID-driven flexibility and suggests that a culture of resilience in our libraries will help us to more easily adapt to these, and emerging, changes we will inevitably encounter. That is followed by Carole Williams' Public Libraries Leading the Way column, “Delivering: Automated Materials Handling for Staff and Patrons," in which she discusses the effects of an automated materials handling system on both the staff and patrons of the Charleston County (SC) Public Library.

In peer-reviewed content, we have a diverse set of articles on range of topics: bias mitigation in metadata; accessibility of PDF documents; two articles on automated classification of different kinds of texts; two articles with lessons learned due to our abrupt move to remote service; and a case study on the importance of product ownership.

1. Mitigating Bias in Metadata: A Use Case Using Homosaurus Linked Data / Juliet Hardesty and Allison Nolan

2. Accessibility of Tables in PDF Documents: Issues, Challenges and Future Directions / Nosheen Fayyaz, Shah Khusro, and Shakir Ullah

3. Text Analysis and Visualization Research on the Hetu Dangse During the Qing Dynasty of China / Zhiyu Wang, Jingyu Wu, Guang Yu, and Zhiping Song

4. Topic Modeling as a Tool for Analyzing Library Chat Transcripts / HyunSeung Koh and Mark Fienup

5. Expanding and Improving Our Library's Virtual Chat Service: Discovering Best Practices When Demand Increases / Parker Fruehan and Diana Hellyar

6. A Rapid Implementation of a Reserve Reading List Solution in Response to the COVID-19 Pandemic / Matthew Black and Susan Powelson

7. Product Ownership of a Legacy Institutional Repository: A Case Study on Revitalizing an Aging Service / Mikala Narlock and Don Brower

Kenneth J. Varnum, Editor

varnum@umich.edu

September 2021 